\title{
Implementation of spigot discharge systems for high-density tailings at Sierra Gorda Sociedad Contractual Minera, Chile
}

\author{
J Engels Tailpro Consulting, Chile \\ H Gonzalez Sierra Gorda Sociedad Contractual Minera, Chile \\ G Aedo Sierra Gorda Sociedad Contractual Minera, Chile \\ GI McPhail Water, Waste and Land Australia Pty Ltd, Australia
}

\begin{abstract}
During 2016, Sierra Gorda Sociedad Contractual Minera (SCM) completed a concept study for the modification of their single point tailings discharge system to a spigot style arrangement with the goal of increasing beach slope and increasing the deposition area within their tailings deposit limits. A series of deposition trials on a laboratory scale were completed to identify design and operational parameters associated with implementing spigot style systems. Deposition modelling was also completed to assist with the development of the short and long-term design for the tailings storage facility.

During 2017, a trial spigot system was developed operating gravitationally for 58-62\% solids for up to $110,000 \mathrm{tpd}$. Due to the success of this system, this was subsequently complemented with three additional spigot systems with a further four to be developed in 2018.

This paper provides a review of the laboratory through to industrial-scale trials conducted and the results and optimisations considered for the short and long-term design of Sierra Gorda SCM's tailings storage facility.
\end{abstract}

Keywords: beach slope, laboratory, industrial trial, spigots

\section{$1 \quad$ Introduction and objectives}

\subsection{Project overview}

Sierra Gorda Sociedad Contractual Minera (SCM) (KGHM International Ltd (55\%), Sumitomo Metal Mining $(31.5 \%)$ and Sumitomo Corporation (13.5\%)), is a copper, gold and molybdenum mine operation located approximately $60 \mathrm{~km}$ from the city of Calama in Northern Chile (II Antofagasta Region). The mine has been operating since 2014 , with proven and probable ore reserves of $800 \mathrm{M} \mathrm{t}$ containing grades of $0.35 \%$ copper, $0.04 \mathrm{~g} / \mathrm{t}$ gold and $0.01 \%$ molybdenum. Current operations are 110,000 tpd (increasing to 230,000 tpd in Phase II) with the produced copper and molybdenum concentrate being sent by train to Sierra Gorda SCM's port facilities located in Antofagasta. Sierra Gorda SCM operates with the use of sea water from the cooling systems of a power plant in the town of Mejillones. Instead of being recycled to the sea, the water is pumped to the processing plant through a $144 \mathrm{~km}$ pipeline located at an elevation of 1,700 $\mathrm{m}$ above sea level.

\section{$1.2 \quad$ Tailings storage}

As part of the design of Sierra Gorda SCM, high-density thickened tailings (HDTT) discharge was considered. The original tailings storage facility (TSF) design considered discharge of tailings at $62 \%$ solids $(\mathrm{w} / \mathrm{w})$, having an average achievable beach slope of $1 \%$ and a storage capacity of 1,350 $\mathrm{M}$ t of tailings over the 20 year life of the operation. During commissioning of the processing plant in 2014, the design solids concentration of the tailings was not consistently achieved by the two $86 \mathrm{~m}$ diameter high-rate tailings thickeners. The principal reasons relate to the variations in particle size distribution of the current tailings, which are slightly 
finer, and the torque limitations of the thickeners. Since 2014 to date, tailings are discharged at $58-62 \%$ solids ( $w / w$ ) with beach slopes of $0.3-0.5 \%$ being achieved (via single point discharge).

Until February 2017, the TSF was designed to operate by depositing tailings sub-aerially via single point discharge from predominately near the centre of the 2,300 ha TSF via a $1.2 \mathrm{~m}$ diameter, $2.4 \mathrm{~km}$ long pipeline from the processing plant. A second single point discharge was used to an emergency area nearer to the processing plant. The design of the tailings transport system considers gravitational discharge from the main underflow starter box adjacent to the two tailings thickeners. The only thickened tailings pumps considered in the design are located below the tailings thickeners reporting to the starter box initiating $100 \%$ gravitational transport of tailings to the TSF.

Operation of the single point discharge system has resulted in the tailings flowing as single channels down to the main embankments of the TSF without developing a beach over the natural ground (Figure 1). The reasons primarily being that the tailings formed a flatter beach than expected, less than the natural ground slope. The tailings therefore accumulated against the main confining embankment and started to eventually develop a beach in the lower area of the TSF for approximately 2.5 years of operation accelerating the reduction in freeboard of the starter embankments, thus requiring increased CAPEX to develop larger retaining embankments in the short to medium-term. As a consequence of this, a change in the deposition design was recommended and implemented by Tailpro Consulting, Water, Waste and Land Australia and Paterson \& Cooke in February 2017.

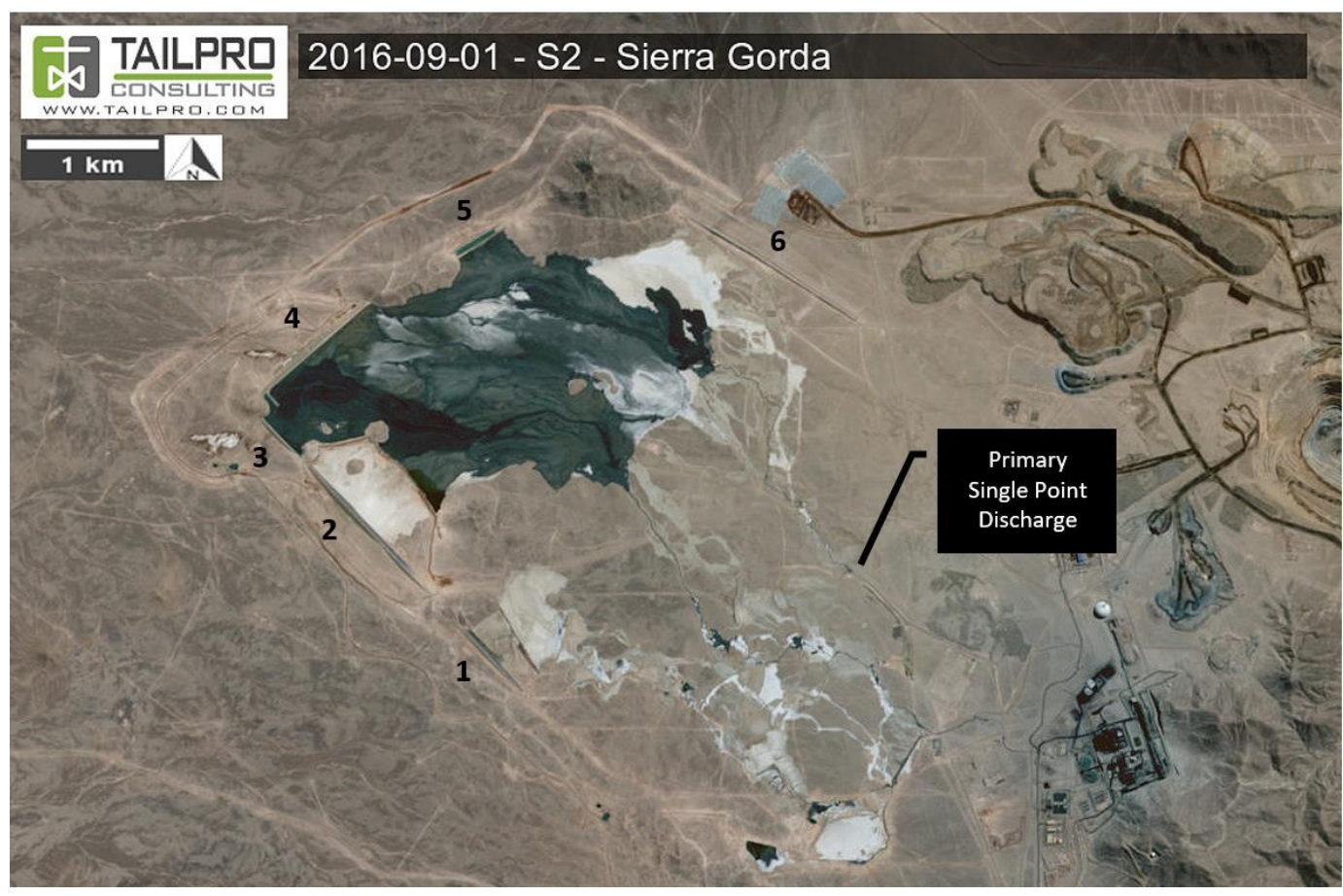

Figure 1 General layout of the Sierra Gorda SCM TSF (main embankments numbered)

\subsection{Developments and objectives}

A series of studies relating to the deposition of tailings was carried out in 2016 with the goal to try and increase the beach slope considering the current tailings (58-62\% solids). Conceptual layout designs and hydraulic calculations were made to ensure the existing tailings distribution system could be modified to increase the beach slope when considering the significant limitation associated with a gravitational thickened tailings delivery system.

This paper provides a review of the laboratory through to industrial-scale trials conducted and the results and optimisations considered for the short and long-term design of Sierra Gorda SCM's TSF. 


\section{Conceptual study}

Following an initial visit by Tailpro Consulting to Sierra Gorda SCM in early 2016, observations were made to potentially improve the discharge planning and operation of the TSF with the goal to stack tailings over the natural ground. Samples were consequently sourced from the main single point discharge and laboratory flume trials were conducted to define beaching-related rheology for application in a preliminary beach slope model for the Sierra Gorda SCM TSF. A range of concentrations of solids (55-62\% solids) was considered to assess the likely impact of the rheology of the tailings on the beach predictions.

\subsection{Laboratory tests}

A flume rheometer has been used to determine the low shear rate rheology for the Dr Gordon McPhail beach slope model (McPhail 1995, 2008) that has been used at the adjacent Minera Centinela mine for a similar scale operation as well as subsequent design of their current tailings distribution system. Based on the experience gained at Minera Centinela (Gaete 2014; Gaete et al. 2014; McPhail et al. 2017), and considering the similar throughput and tailings properties to Sierra Gorda SCM, the McPhail beach slope model was considered to be the most reliable methodology with which to assess the proposed modifications to the tailings transport design and distribution systems.

The flume rheometer developed by McPhail (2014) requires a constant level of tailings (fully sheared) within its header tank to maintain a steady flow rate into the flume (Figure 2). Velocity and elevation change of the advancing tailings are recorded automatically during discharge. After the test, the depth of the deposited tailings is exported to a computer model and samples are taken to determine the percent solids and the rheology of the slurry along the flume. The test is repeated at four different flow rates for each solids concentration by re-using the fully sheared slurry.

For the rheological assessment, two samples were considered representing the upper and lower bounds of particle size distribution for the actual and near future plant conditions to assess variability.

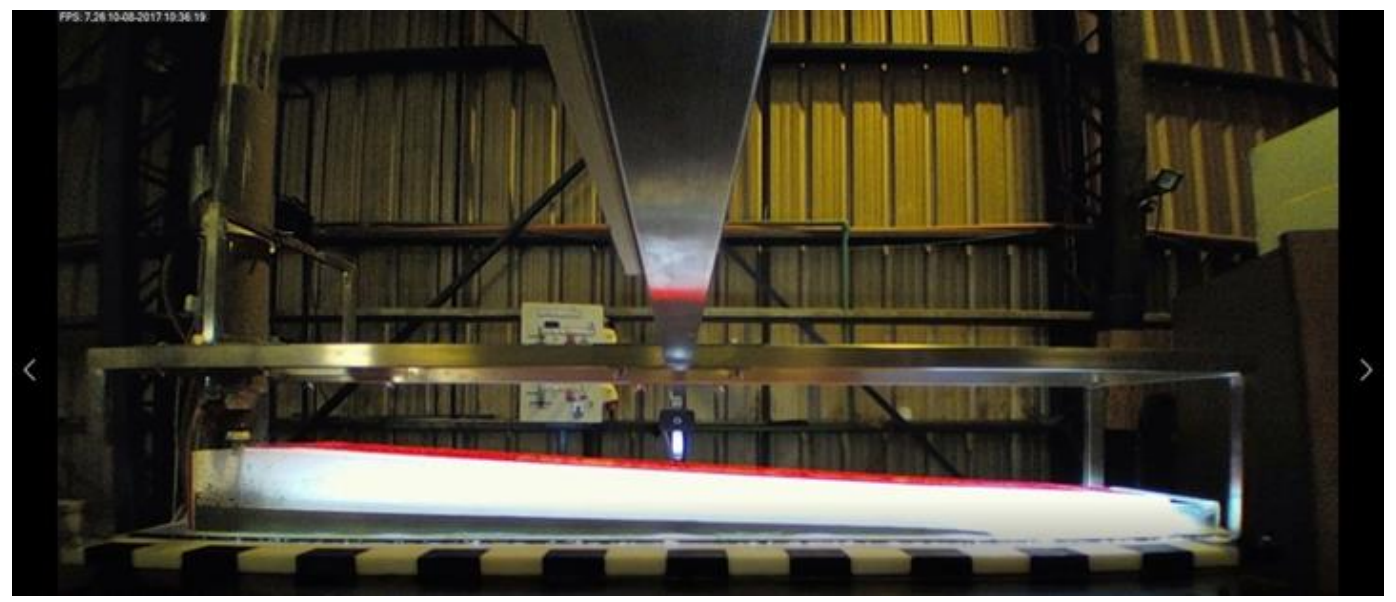

Figure 2 Laboratory deposition trials (March 2016)

The results were analysed using continuity, force-momentum flux, and Bernoulli to calculate the shear stresses along the base and sides of the flume. From these shear stresses an estimate of the flow curve at low shear rate can be made. The focus of these flume rheometer tests is to determine rheological parameters under settling conditions as the tailings flow rather than try and measure a comparison of beaching slope between different solids concentrations (as is common with most small-scale flume trials today).

Data from the flume rheometer tests were evaluated and interpreted to derive representative estimates of the beaching-related rheological parameters of the slurry. This information was used to determine the optimum number and spacing of spigots required to stack the tailings over the natural ground slope. 


\subsection{Conceptual engineering}

The focus of the conceptual engineering study was to determine the feasibility of modifying the existing single point deposition system to a spigot style design and operational strategy to steepen the beach slope. The largest limitation is the available pressure at the spigots since this is controlled by gravity. This complicates the distribution of tailings, especially during flow fluctuations from the thickeners.

For the conceptual study, two options were investigated to develop a short to medium-term design philosophy for Sierra Gorda SCM:

- Option 1 contemplated a review of the gravitational tailings system to the TSF and the optimised distribution of the tailings via spigots in the upper area of the TSF. The tie-in to the existing system was key to maximising the gravitational head available.

- Option 2 contemplated continuation of the design of Option 1, but prolonging the life of the gravitational system by considering a tie-in above the primary starter box to gain elevation and, therefore, greater energy. This option was considered at a conceptual level to assess the possibility of delaying the pumping system to form part of Phase II of the project.

To generate a beach slope steeper than the ground slope, beach modelling indicated that it would be necessary to limit the flow rates through the spigots to $60 \%$ of the total flow rate discharged through 12 spigots referred to as Type A spigots. The remaining $40 \%$ would need to be discharged through four spigots referred to as Type B spigots located at the ends of the spigot distribution bars. The spigot arrangement, spigot distribution bar and delivery pipe arrangement is indicated in Figure 3. The net effect of this arrangement would be a bi-slope beach with the first half of the beach length dominated by the beaching from the Type A spigots producing a slope of $1.6 \%$ and the second half of the beach length dominated by the Type B spigots producing a slope of $0.5 \%$.

Phase I of the project makes maximum use of gravitational discharge. Phase II of the project considers installing a pumped tailings distribution system due to be implemented in 2024 to allow the spigots to gain elevation.

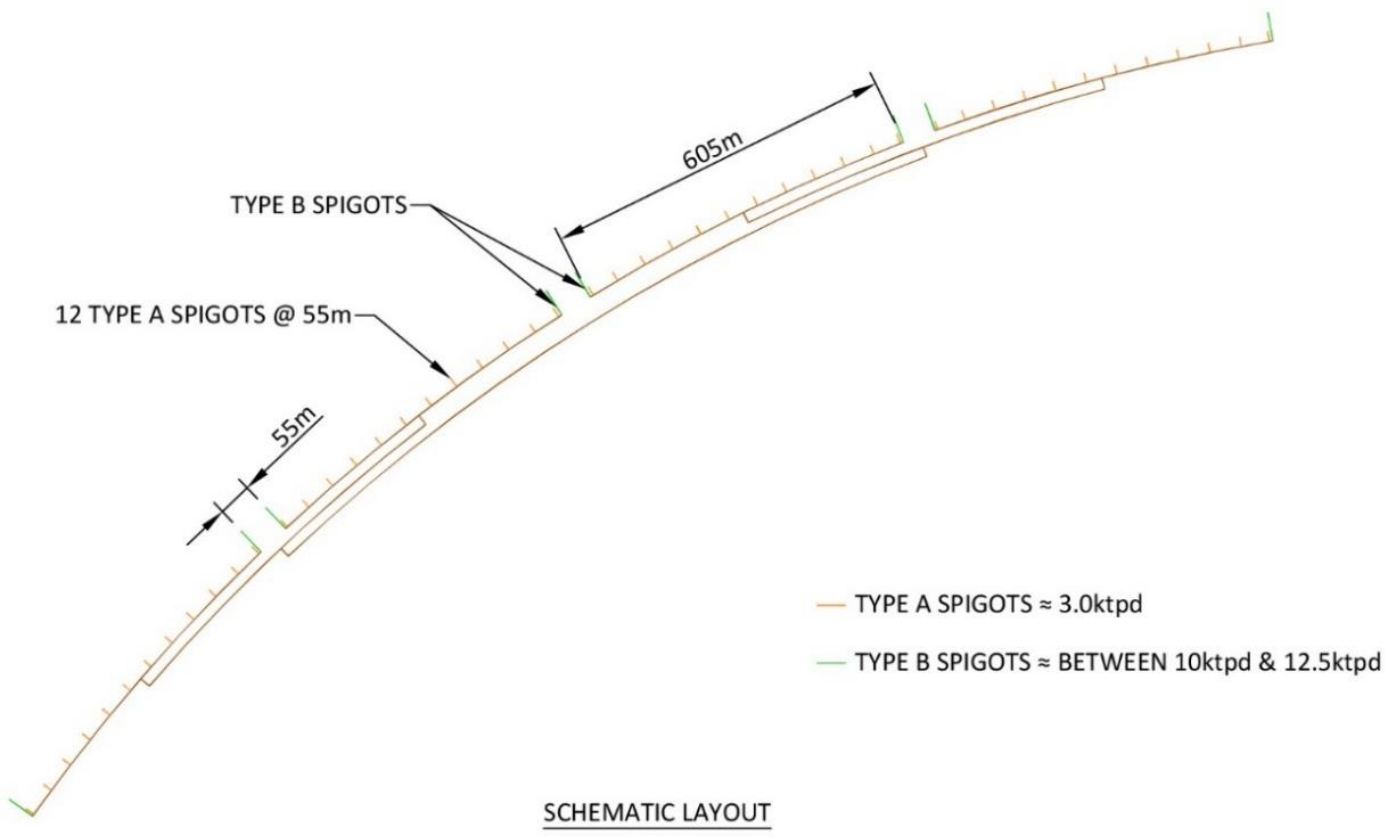

Figure 3 Schematic layout of the four distribution bars and location of the spigots (Type A and Type B)

Another important factor in the design was the lack of water pressure available to flush the distribution bars and spigots following active discharge. This meant that the system had to auto-drain during shutdowns and had to have tight control of the plant tonnage to ensure the system does not sand and block. 
The following filling plan (Figures 4 and 5) was considered on a conceptual level where an average beach slope of $1.6 \%$ was estimated from the beach modelling of the Type A spigots ( $0.5 \%$ from the Type B) and spigot operation plans.

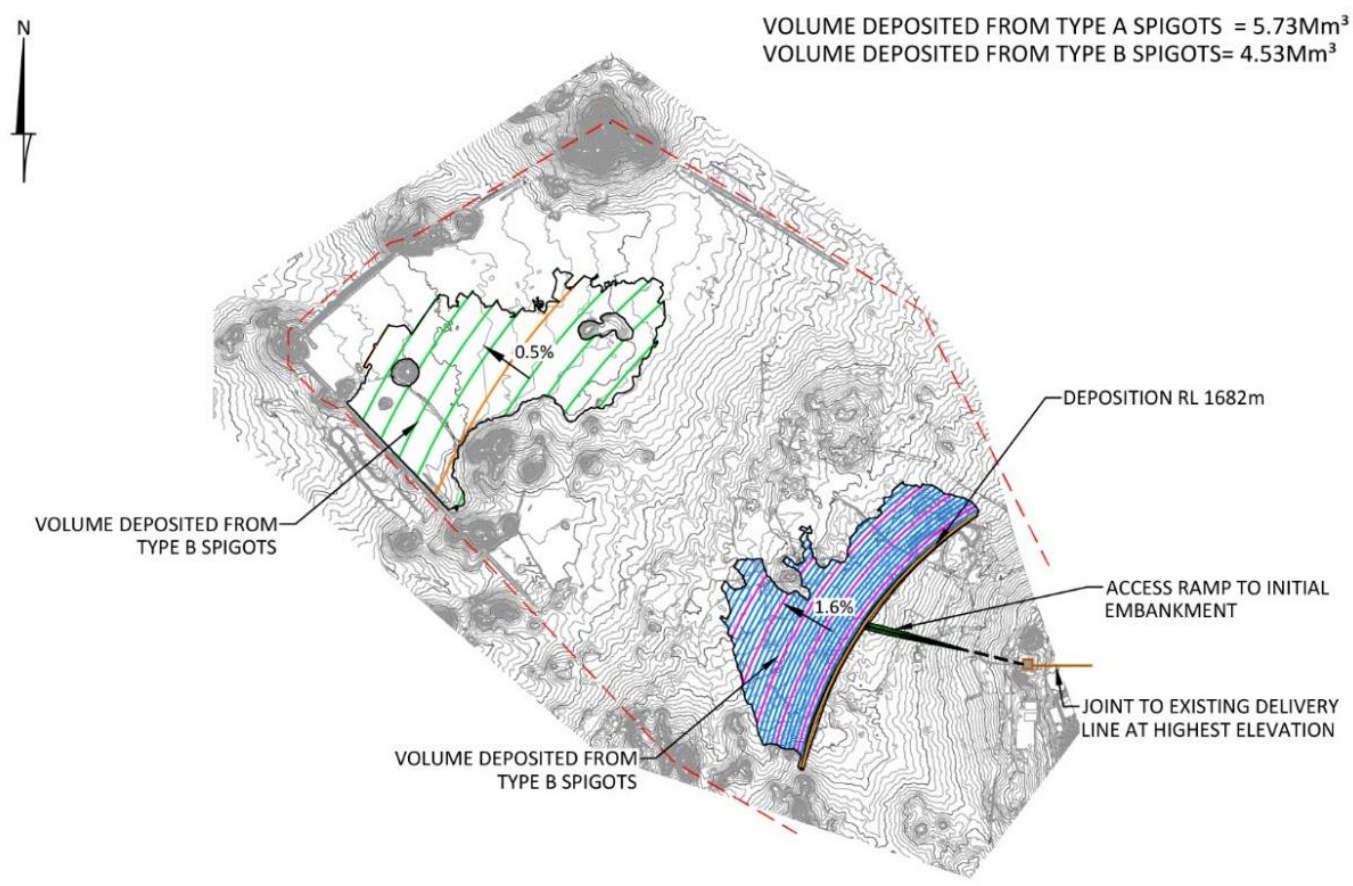

Figure 4 Plan of TSF showing deposition to an elevation of RL 1,682 $\mathrm{m}$ at the spigots

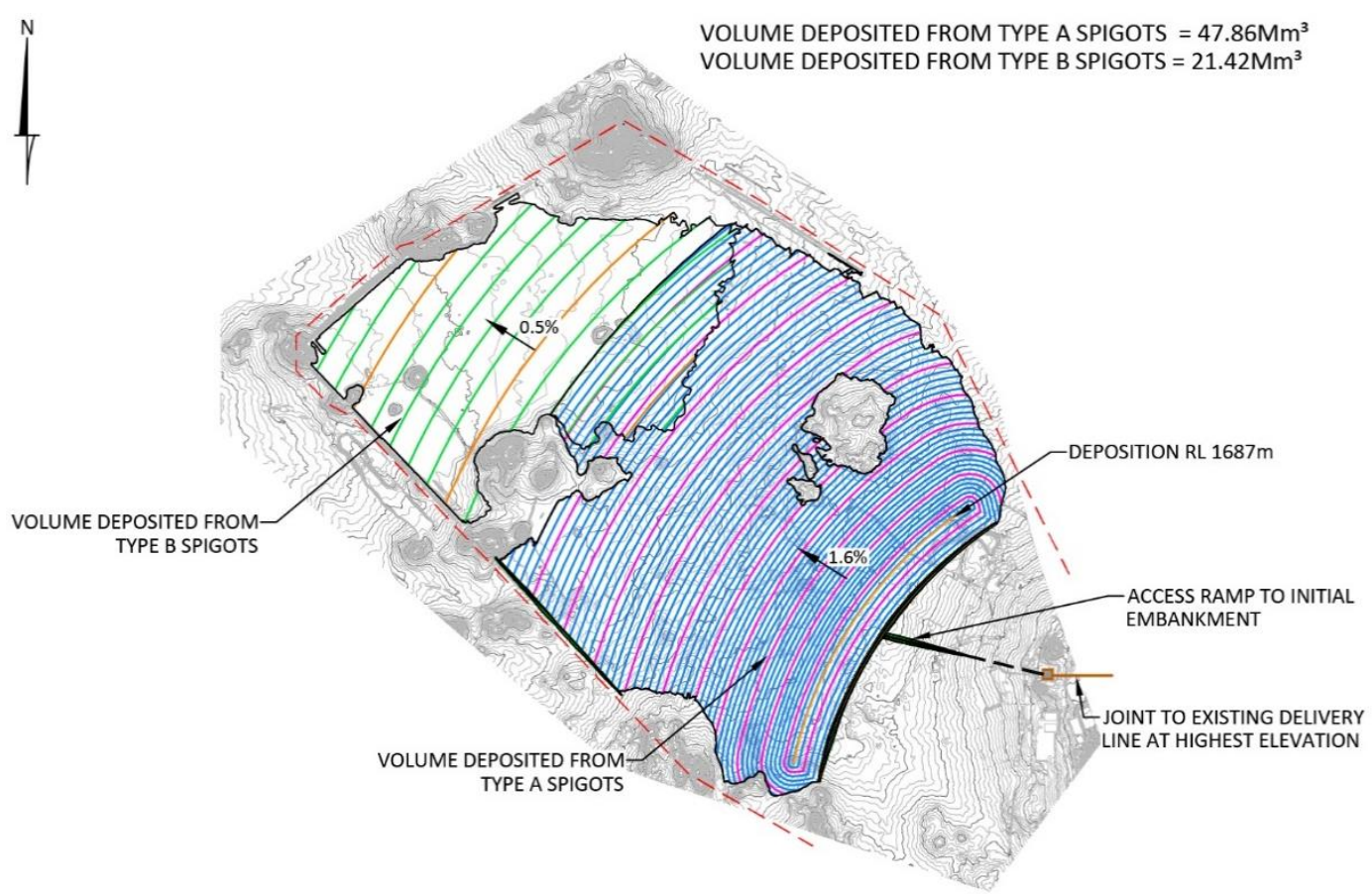

Figure 5 Plan of TSF showing accumulated deposition to an elevation of RL 1,687 $\mathrm{m}$ at the spigots 
A starter embankment was constructed to place the various distribution bars with the spigots having slotted drop-bars to prevent scour of the embankment. The slots close naturally as the level of the deposited tailings rises. This is shown in the top schematic in Figure 6.

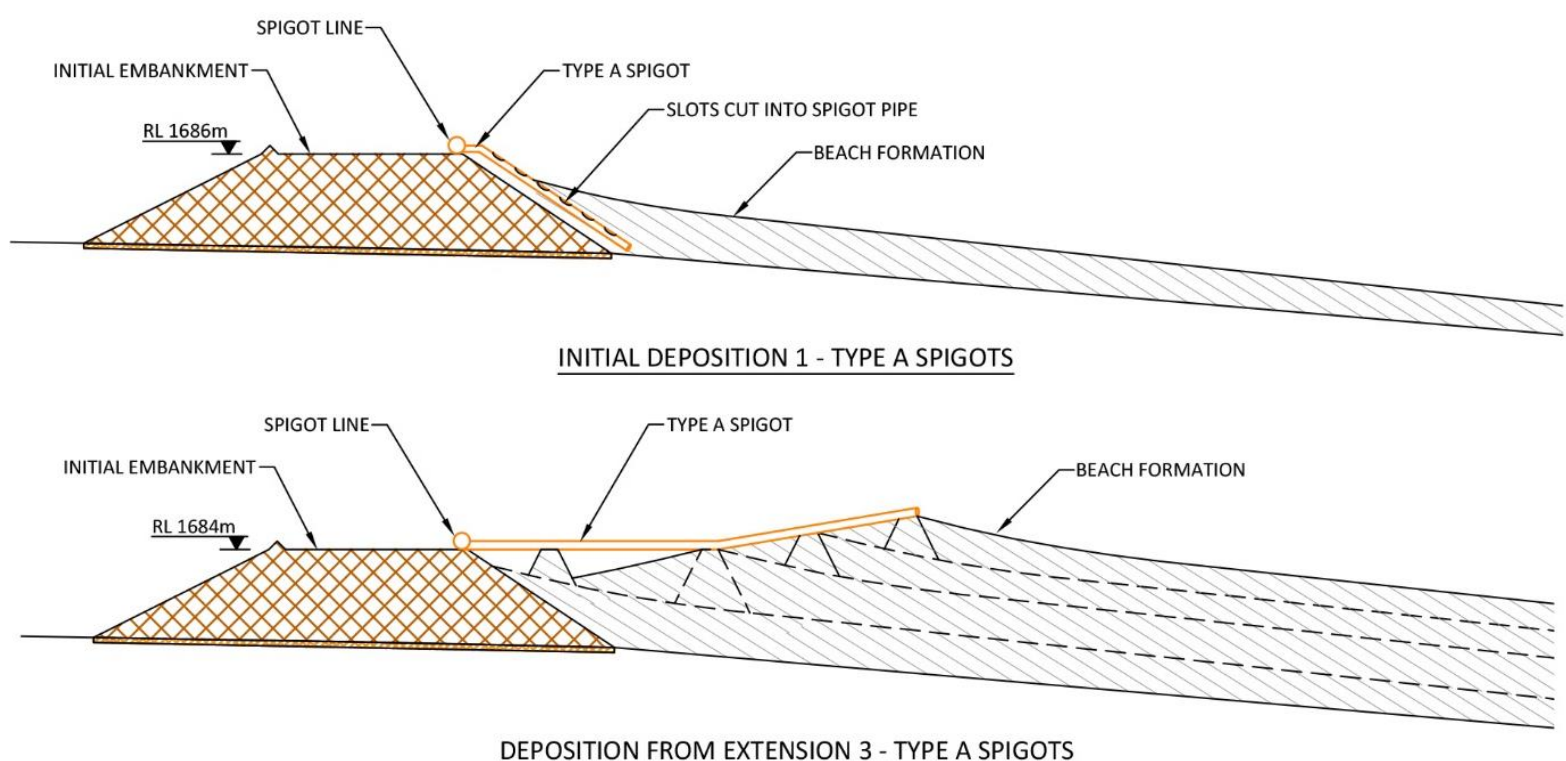

DEPOSITION FROM EXTENSION 3 - TYPE A SPIGOTS

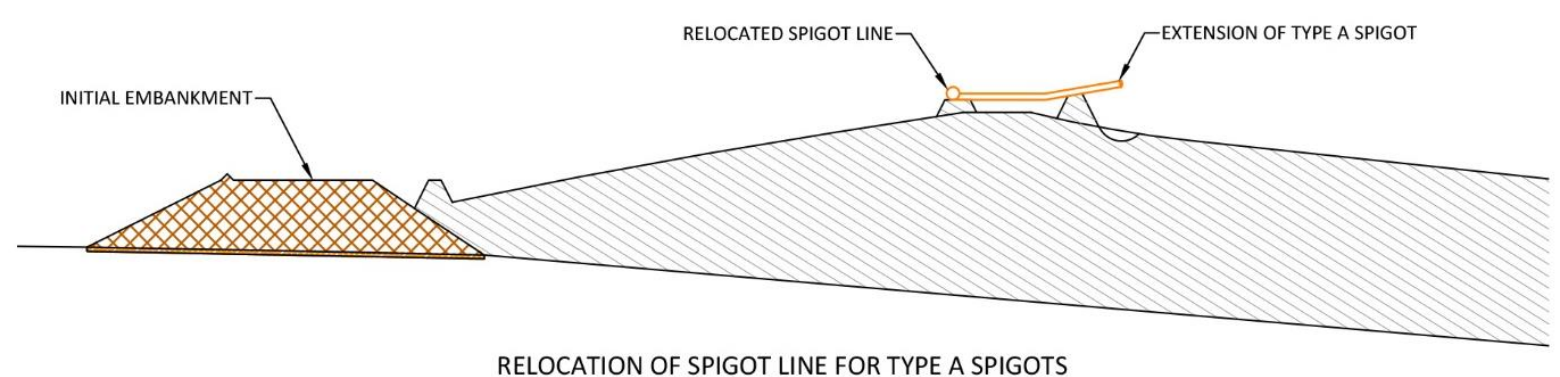

Figure 6 Spigot Type A - advance and follow technique (vertical scale exaggerated)

The gain in elevation of the spigots was intended to be based on the advance and follow technique, whereby the spigots progressively advance considering extensions as the beach rises as indicated in the middle schematic in Figure 6. After a specified number of advances, the main distribution bar is relocated at the crest and the spigot advancement process restarts. Volume gain was considered by incrementally raising the discharge outfalls as new advances are made. An elevation of $\mathrm{RL} 1,687 \mathrm{~m}$ was determined to be the limit of the spigot elevations based on the existing gravitational system. Option 2, as discussed previously, would be required to gain further head to increase the discharge elevation and later for Phase II, the installation of a pumped system.

\section{Industrial-scale trial}

Towards the end of 2016, Sierra Gorda SCM decided to install a trial spigot distribution bar that could be later integrated into the full proposed system. This trial spigot distribution bar, named S1, was to receive $50 \%$ of the plant production with the other $50 \%$ sent via a direct outfall to an adjacent area of the TSF, away from the S1 beach formation area. The reason for the trial was to confirm to Sierra Gorda SCM the benefit of beach slope formation, reduce initial investment and risk of installing spigots, and allow for modifications to the subsequent distribution bars if required. 
The trial distribution bar was commissioned in early February 2017. Figure 7 shows a satellite image before (left) and shortly after commissioning (right) of the S1 spigots showing tailings depositing over virgin ground and filling shallow valleys.
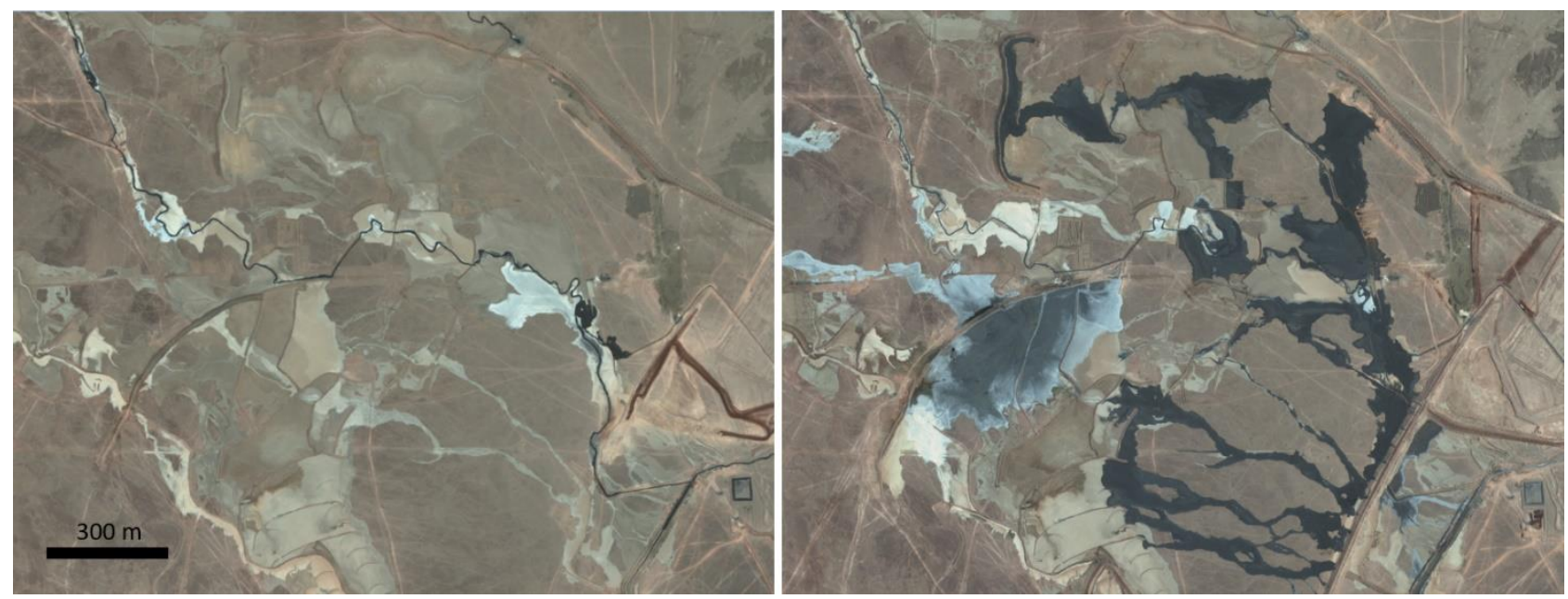

Figure 7 Satellite images prior to and post commissioning of the S1 spigots

The trial was a success with only minor blockages occurring at the extremes of the distribution bar (last Type A and Type B spigots). This was due to a combination of lack of flushing water pressure and drainage of the spigots. Minor changes to valve specifications, local emergency flushing points and improved spigot slot designs were considered for the development of the other distribution bars.

Due to the success of the trial, another two distribution bars were developed shortly after the installation of S1 and subsequently commissioned in July 2017. These bars, N1 and N2, would operate alternately with the S1 and a new S2 distribution bar to the south.

The S1 distribution bar operated independently until October 2017 when the $50 \%$ direct discharge was connected to distribution bar S2, a symmetrical layout of S1, and full plant flow discharged $100 \%$ via the spigot systems (as per the original concept presented in Figure 3).

\section{Development of the long-term distribution system}

The goal of Sierra Gorda SCM's tailings management strategy is to not only stack the tailings via the spigot distribution system, but also to optimise the in situ density and capacity of the TSF reducing the elevations of the large perimeter walls at final capacity. In the short-term, and due to the reduced freeboard during 2017 , no tailings were to be discharged towards the main retaining embankments so as to allow the already deposited tailings to dry and consolidate as well as allow time to install underdrain extensions and subsequent raising of the main retaining embankments. During this time, temporary internal embankments were constructed to store tailings while the various spigot systems were commissioned. These internal embankments are considered temporary in that they will eventually be covered by tailings discharged from the spigots. Interwall drop pipes were considered to transfer flow from one side of an embankment to the other lower side thereby enabling controlled filling of the TSF as the beach from the spigots developed.

In early 2018, the main embankment walls had been raised and tailings discharge to this area was resumed. This area was principally considered for storage of tailings from the Type B spigots as well as direct discharge of tailings when the main spigot systems could not be operated (e.g. low tonnage from the processing plant or low solids concentrations of tailings). 


\subsection{N1 and N2, and S1 and S2}

\subsubsection{Overview}

The first spigot systems to be developed were located in the upper area of the TSF, as per the original concepts, and were intended to operate until the end of mine life in 2041 (Figure 8). The installation and operation of these spigots would imply that the concept of changing the beach slope to stack over natural ground would increase the active area of the TSF allowing thinner layers to be deposited, thus increasing the in situ density of the tailings. Reducing the main downstream perimeter wall heights was also an advantage but required that some of the upstream perimeter wall construction be brought forward to retain the tailings within the TSF limits as they stack in the upper area of the facility.

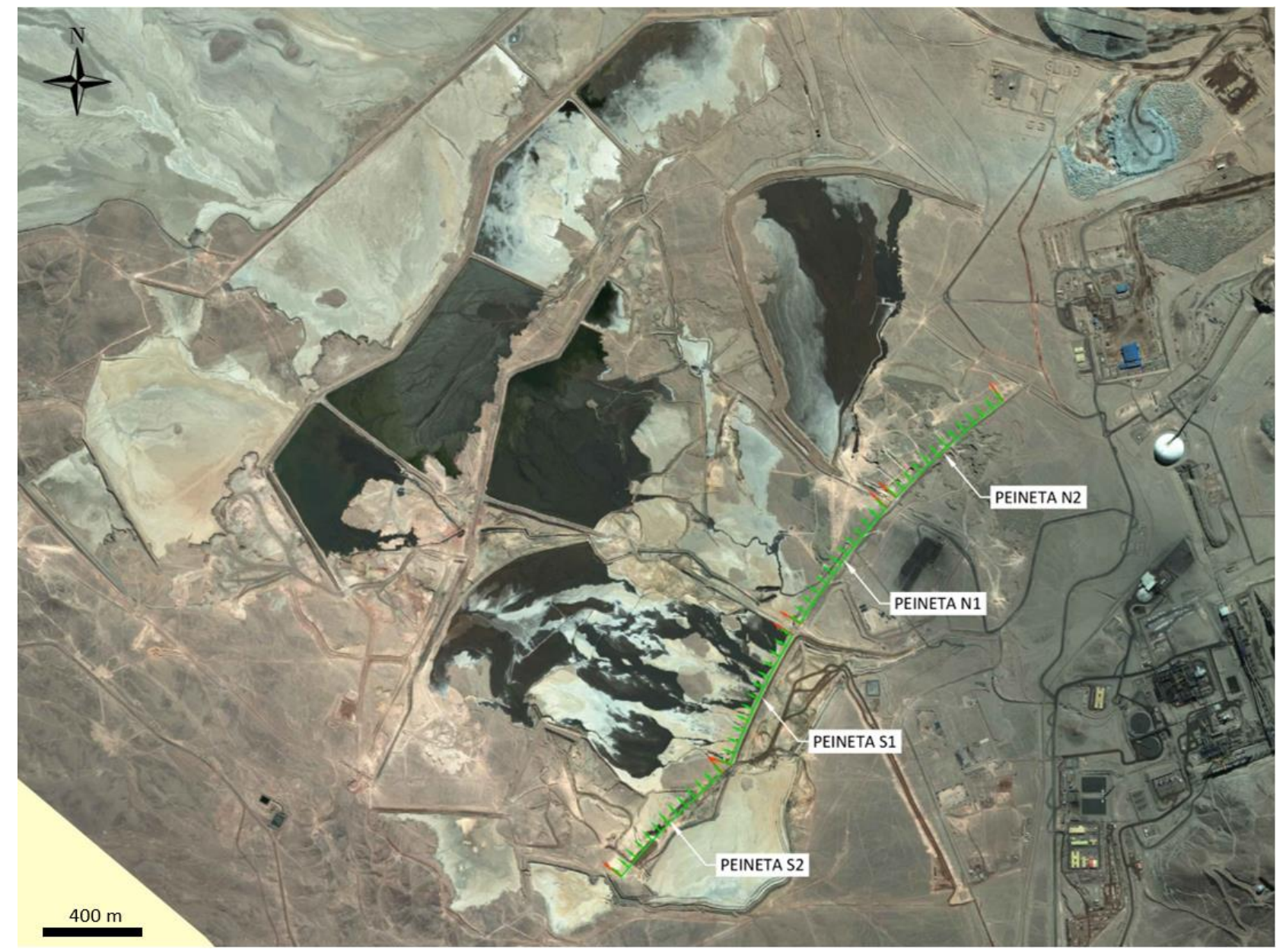

Figure 8 Plan of TSF showing N1 and N2, and S1 and S2 spigot distribution systems

\subsubsection{Development}

To date, the original S1 spigot system has been operating the longest, and the time of writing this paper the beach slope is still continuing to develop and gain gradient. Figure 9 shows a typical section of the S1 beach for various topographical surveys completed between commissioning in February 2017 until September 2017. Immediately downstream of the spigots, beach slopes in excess of $3 \%$ are currently being achieved with an average of approximately $2 \%$ slope over the $1.2 \mathrm{~km}$ beach length down to the lower retaining internal wall. 


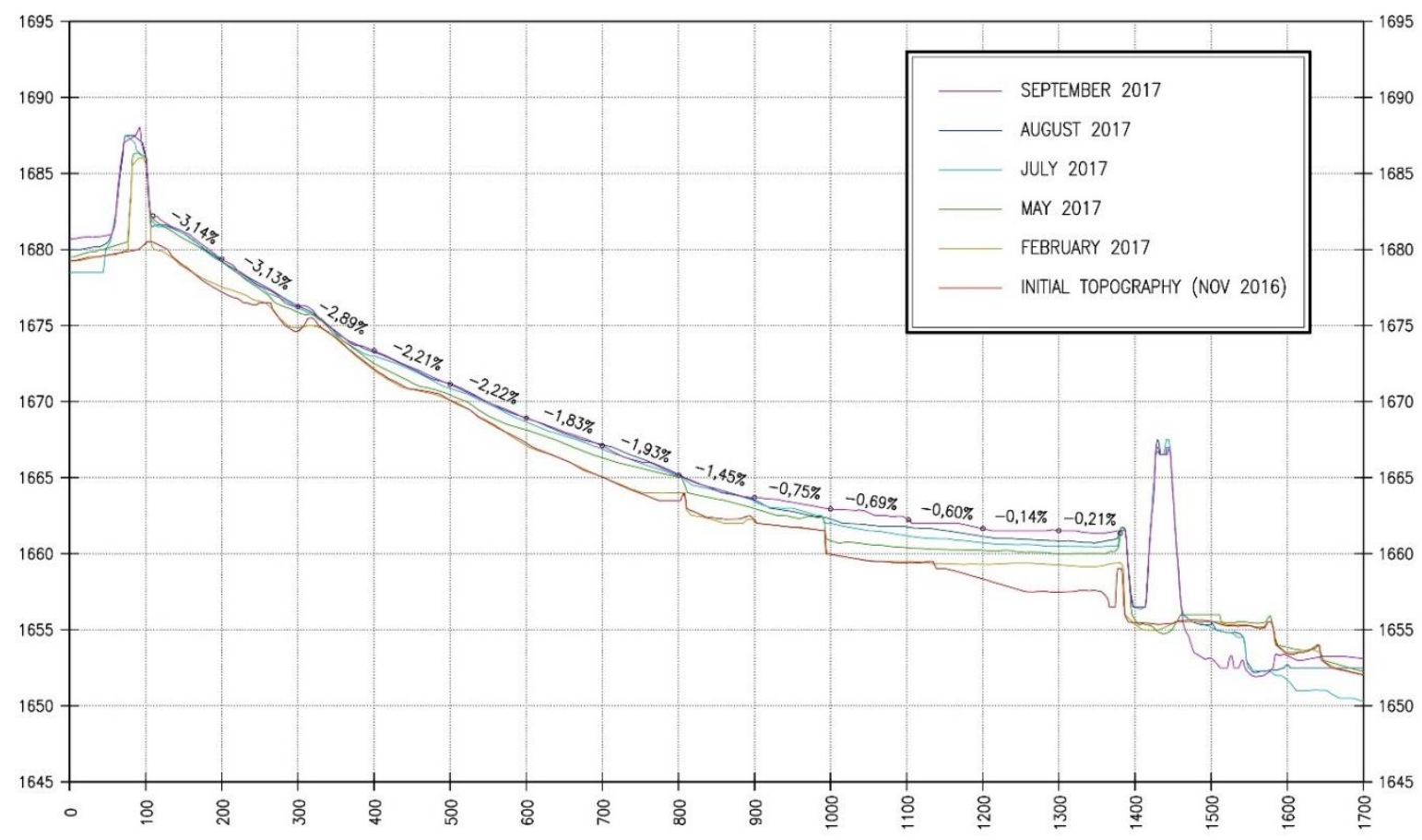

Figure 9 S1 beach development (February to September 2017) - vertical scale twice exaggerated

The beach is formed primarily by the Type A spigots with the Type B spigot flows reporting to the lower retaining wall downstream. The beach slope formed by the Type A spigots has proved to be slightly higher than the predicted $1.6 \%$ and the beach formed by the Type B spigots is also slightly higher than expected, approaching $0.6 \%$. The spigot spacing considered has shown to be adequate and in line with the original independent-flow beach slope predictions.

At the time of writing this paper, the $\mathrm{S} 2$ spigots had only just been commissioned and beach development will be reported during the seminar presentation. Figure 10 shows the spigot beaches forming downstream of each distribution bar as of early October 2017 (S2 not yet commissioned).

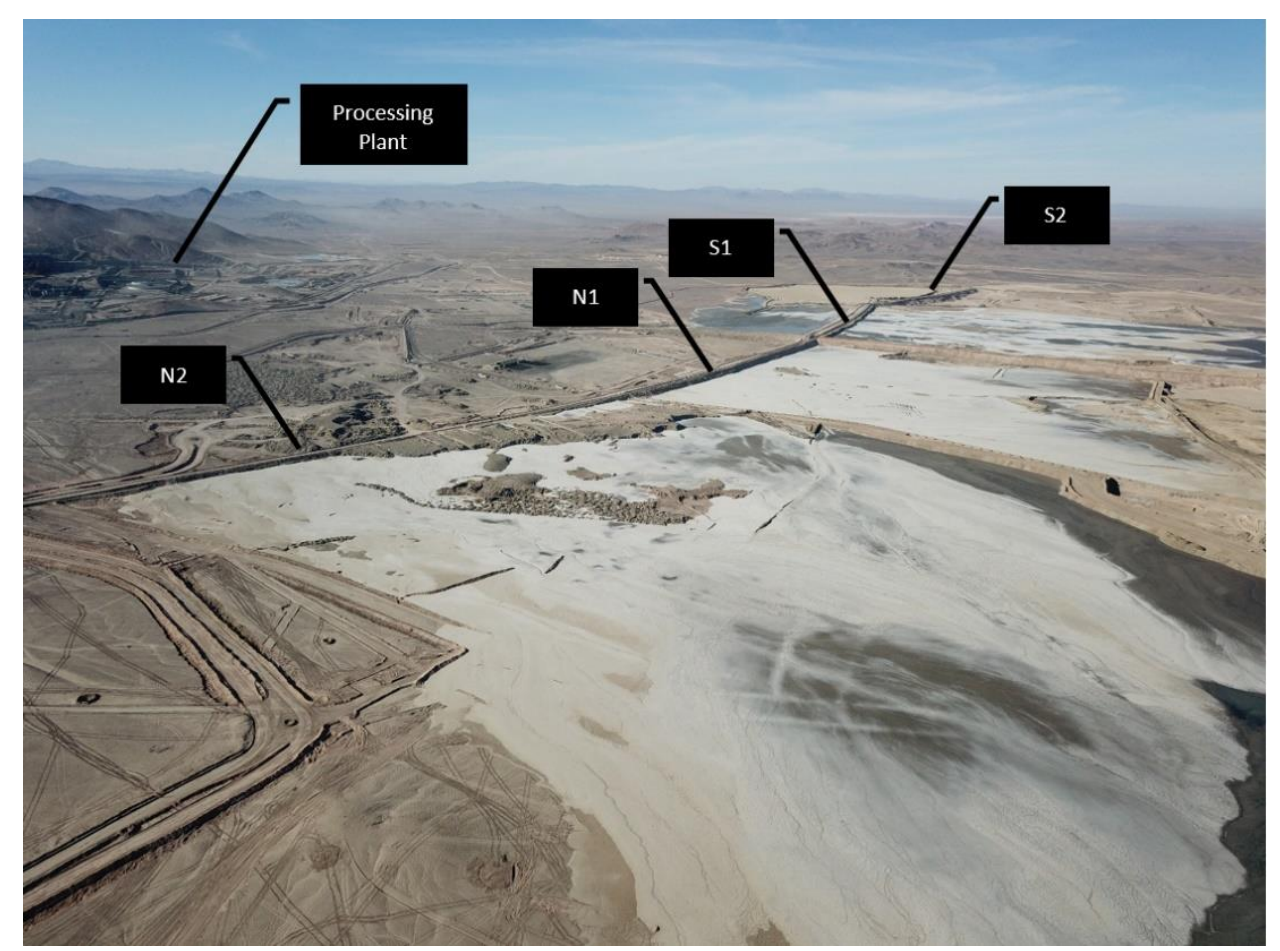

Figure 10 Aerial image of the N1 and N2, and S1 and S2 spigot distribution systems (October 2017) 


\subsection{N3 and N4, and S3 and S4}

\subsubsection{Overview}

A second set of spigot systems are to be implemented to assist with developing additional deposition fronts and provide Sierra Gorda SCM with flexibility in the cyclic discharge and drying of its tailings until the end of mine life. The additional benefit to having an independent spigot system is an opportunity to further reduce the perimeter wall heights by the formation of an intermediate stack towards the middle to lower area of the TSF. This provides an independent spigot front as an adjunct to the operation of the upper area. It was also recommended that the perimeter limits be extended to further increase the beach area development as part of these spigots.

Figure 11 shows the general layout of the N3 and N4, and S3 and S4 spigot systems (together with N1 and $\mathrm{N} 2$, and S1 and S2), and Figure 12 a typical cross-section.

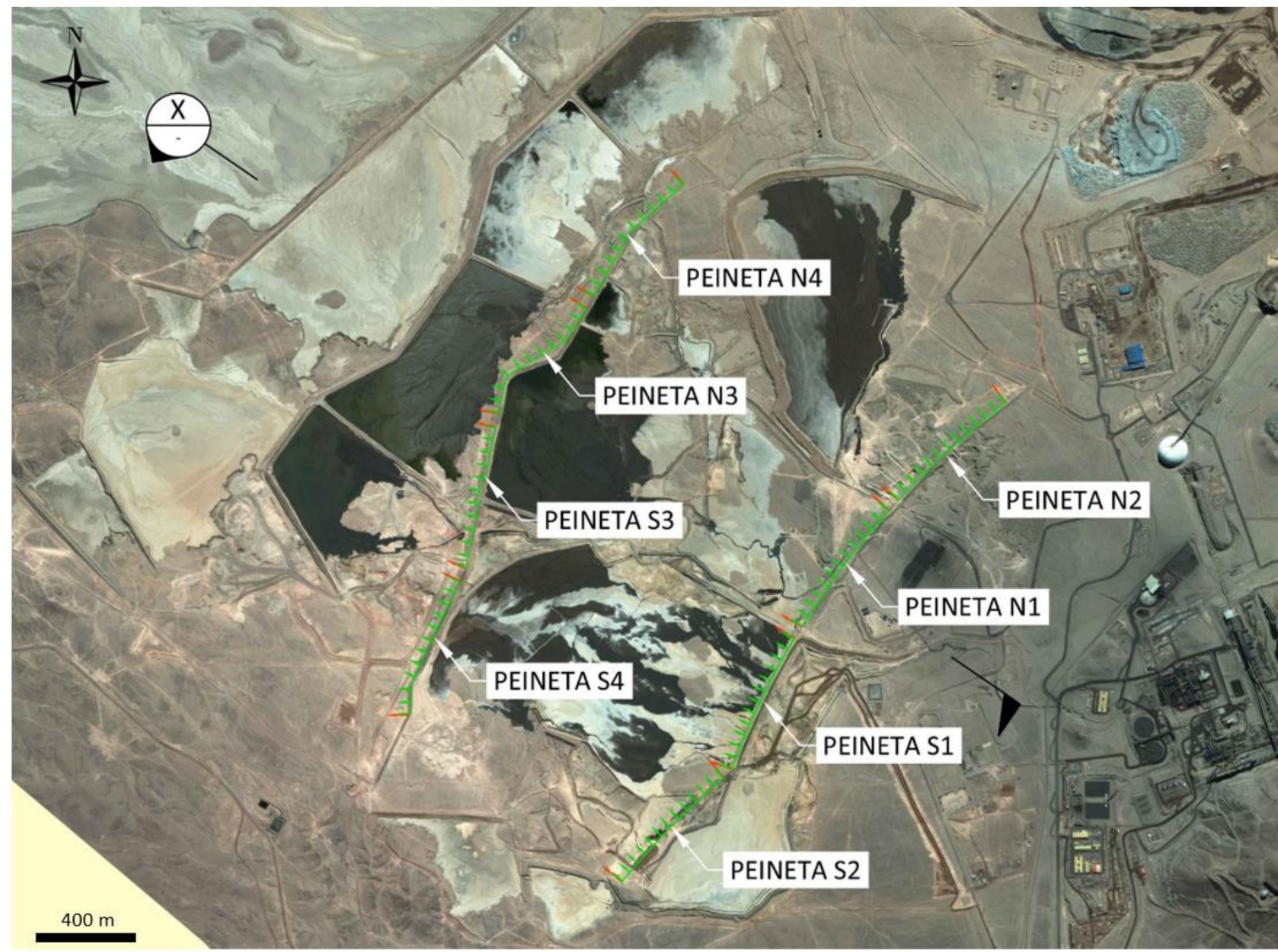

Figure 11 Plan of TSF showing N3 and N4, and S3 and S4 spigot distribution systems

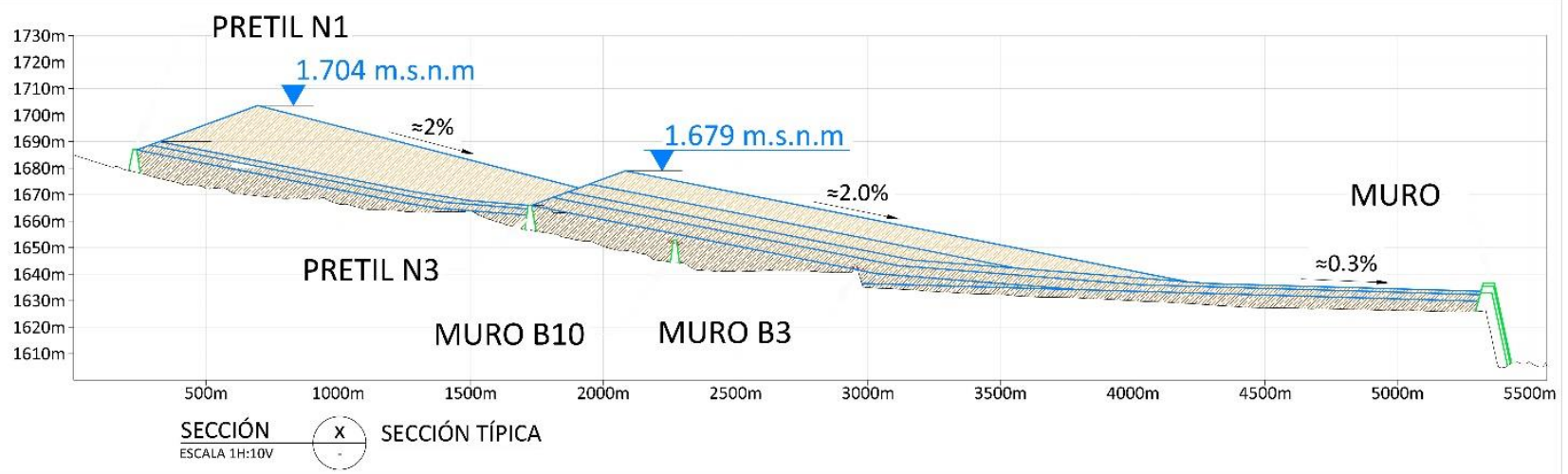

Figure 12 Section through the TSF after commissioning and operating both sets of spigot lines 
At the time of writing this paper, the N3 and N4, and S3 and S4 spigot systems were under construction and were to be commissioned in early 2018. More information will be presented during the seminar presentation.

\section{Conclusion}

The original spigot discharge concepts as developed for Sierra Gorda SCM based on laboratory through to semi-industrial trials have demonstrated that the beach slope can be increased successfully without changing the properties of the tailings. The implementation of the spigots has allowed Sierra Gorda SCM to gain time to develop a more robust medium to long-term tailings storage plan than that based on a lower beach slope as assumed in the original deposition design. Time has also been gained during 2017 to modify and install additional infrastructure and equipment in the main walls while no tailings were deposited upstream.

As part of this study, the knowledge of the tailings properties and their variability proved to be fundamental to be able to provide a spigot design that is adaptable to change. Variations in plant flow rate, solids concentration and rheology have been managed using two spigot types and flow rates to ensure the beach continues to develop and not scour when conditions are unfavourable. Understanding the impacts of the rheology through the lab testing and modelling was essential to reduce risk and provide a more robust design and operational philosophy.

The following tasks are proposed during 2018 to improve the day to day operation of the spigots and provide improved certainty in the medium to long-term design:

- Verification of the predictions and reliability of the concavity of the beach profiles, improvement of the volumetric variations and optimisation of spigot elevations (based on McPhail et al. 2016).

- Analysis of the geotechnical properties of the beached tailings and stability limitations for increasing the beach slope further.

- Optimisation of the perimeter wall heights, layout and design.

- Analysis to optimise the spigot migration to prolong the life of the gravitational discharge system.

- Analysis of an additional thickener to improve the underflow concentration towards the original design target.

- Improvement of the operational philosophy to enhance drying, density and strength gain of the beach.

- Incorporation of closure considerations and landform erosion modelling.

It is envisaged that the spigots will provide Sierra Gorda SCM with a more cost-effective and reliable method to develop their TSF for the life of the project. The capital expenditure associated with the pumped tailings system has successfully been delayed, further justifying the use of the lower spigot system in the short to medium term.

\section{References}

Gaete, S 2014, 'Applying large-scale thickened tailings technology on the sulfide line at Minera Centinela', in S Barrera (ed.), Proceedings of the 2nd International Seminar on Tailings Management, Gecamin, Santiago.

Gaete, S, Bello, F, Engels, J \& McPhail, G 2014, 'Thickening and deposition trials: laboratory through to industrial scale - Minera Esperanza', in RJ Jewell, AB Fourie, PS Wells and D van Zyl (eds), Proceedings of the 17th International Seminar on Paste and Thickened Tailings, InfoMine Inc., Vancouver, pp. 381-391.

McPhail, GI 1995, Prediction of the Beaching Characteristics of Hydraulically Placed Tailings, PhD dissertation, University of the Witwatersrand, Johannesburg.

McPhail, GI 2008, 'Prediction of the beach profile of high-density thickened tailings from rheological and small-scale trial deposition data', in AB Fourie, RJ Jewell, P Slatter and A Paterson (eds), Proceedings of the Eleventh International Seminar on Paste and Thickened Tailings, Australian Centre for Geomechanics, Perth, pp. 179-188.

McPhail, GI 2014 Using small scale flumes to determine rheology at low shear rates, in S Barrera (ed.), Proceedings of the 2nd International Seminar on Tailings Management, Gecamin, Santiago. 
McPhail, GI, Bello, F \& Engels, J 2017, 'Beach profile modelling at Centinela Mine, Chile', Proceedings of the 4th International Seminar on Tailings Management, Gecamin, Santiago.

McPhail, GI, Engels, J \& Ugaz, R 2016, 'Application of fluidised bed column testing to the assessment of beaching and segregation characteristics of mine tailings', in S Barrera and RJ Jewell (eds), Proceedings of the 19th International Seminar on Paste and Thickened Tailings, Gecamin, Santiago, chapter 6, paper 6. 